EPJ Web of Conferences 16, 03001 (2011)

DOI: $10.1051 /$ epjconf/20111603001

(C) Owned by the authors, published by EDP Sciences, 2011

\title{
High performance coronagraphy for direct imaging of exoplanets
}

O. Guyon ${ }^{1,2}$

\author{
${ }^{1}$ Subaru Telescope, National Astronomical Observatory of Japan, 640 N. A'ohoku Pl., Hilo, \\ HI 96720, USA \\ ${ }^{2}$ Steward Observatory, University of Arizona, 933 N. Cherry Ave., Tucson, AZ 85721, USA
}

\begin{abstract}
Coronagraphy has recently been an extremely active field of research, with several high performance concepts proposed, and several new coronagraphs tested in laboratories and telescopes. Coronagraph concepts can be grouped in a few broad categories: Lyot-type coronagraphs, pupil apodization and nulling interferometers. Among existing coronagraph concepts, several approach the fundamental performance limit imposed by the physical nature of light. To achieve their full potential, coronagraphs require exquisite wavefront control and calibration. This has been, and still is, the main bottleneck for the scientifically productive use of coronagraphs on ground-based telescopes. New and promising wavefront sensing techniques suitable for high contrast imaging have however been developed in the last few years and are started to be realized in laboratories. I will review some of these enabling technologies, and show that coronagraphs are now ready for "prime time" on existing and future telescopes.
\end{abstract}

\section{INTRODUCTION}

In the last two decades, technological advances have enabled the detection of hundreds of exoplanets, with an ever-increasing discovery pace [1]. While early exoplanet discoveries were gas giants, rocky planets are now routinely identified. Almost all of these detections are performed with indirect detection techniques such as radial velocity or transit photometry, offering access to orbital parameters, mass, and radius. In a few favorable transiting systems or close-in orbits, low-resolution spectroscopy of exoplanets atmospheres is possible (in transmission for primary transits and in reflection or emission for secondary transits).

Direct imaging of planetary systems is a powerful way to characterize both the planetary system as a whole and its planets. With direct imaging, strong constraints are placed on planetary orbits, planet sizes (from photometry) and planetary atmospheres. Extensive characterization of exoplanets in intermediate to wide orbits (more than approximately $0.1 \mathrm{AU}$ ) does require direct imaging, as their light must be separated from the much brighter starlight in order to perform photometry, spectroscopy, and polarimetry of the planet's light at several points along its orbit. The long-term goal of identifying signs of biological activity in the spectra of exoplanets requires good signal-to-noise (SNR) spectroscopy of intrisically faint exoplanets, and the planet light must be optically isolated from the bright starlight to achieve the required SNR in reasonable exposure times.

In this paper, coronagraph techologies are briefly reviewed. Section 2 gives an overview of coronagraphic approaches, and shows that the large number of existing coronagraph concepts can be grouped in a few large families. The performance of coronagraphs is discussed in section 3 in the absence of wavefront errors or manufacturing defects, and is linked to science requirements for both ground-based and space-based missions/projects.

This is an Open Access article distributed under the terms of the Creative Commons Attribution-Noncommercial License 3.0, which permits unrestricted use, distribution, and reproduction in any noncommercial medium, provided the original work is properly cited. 


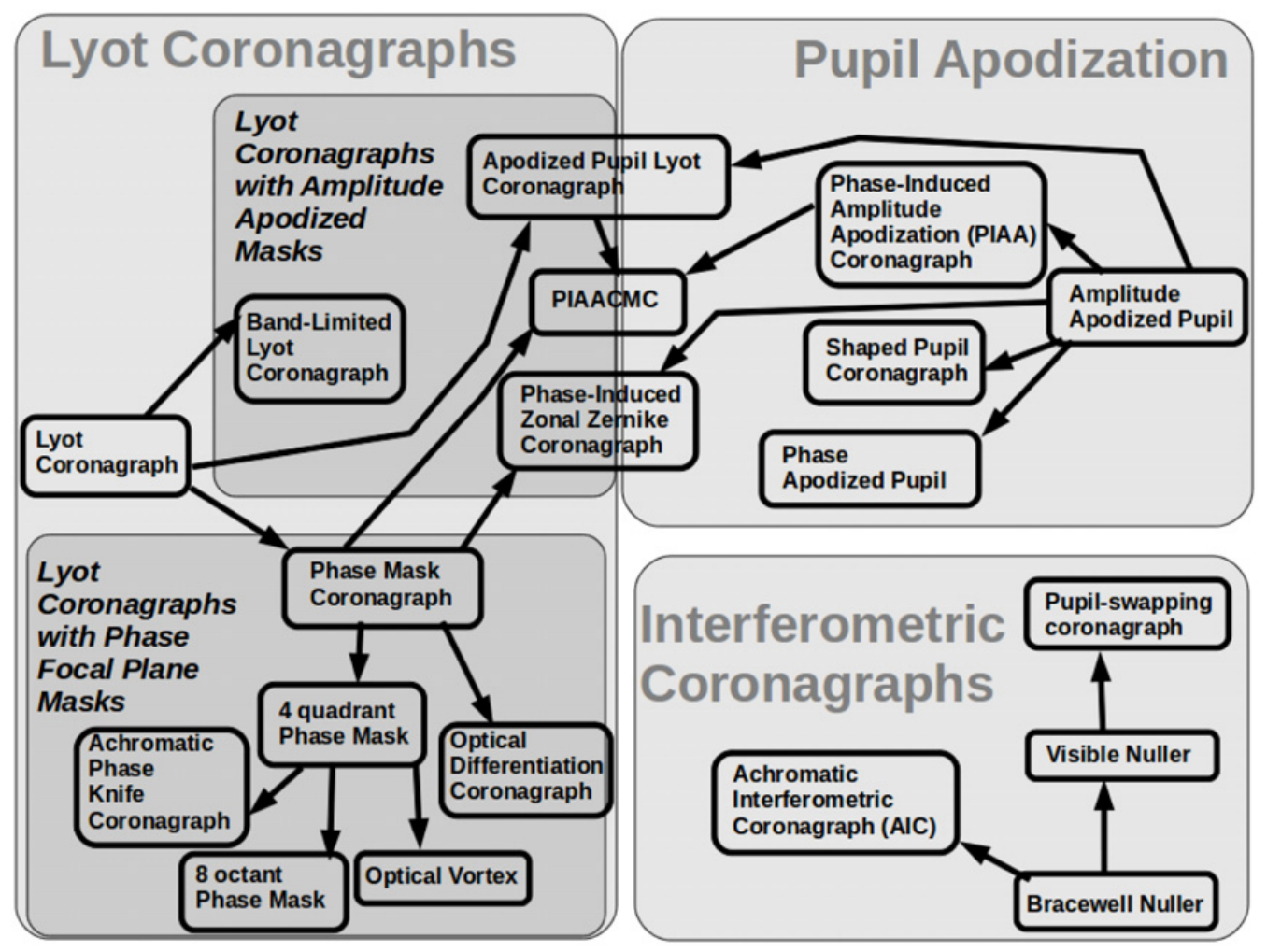

Figure 1. Coronagraphs can be grouped in three broad categories: coronagraphs derived from the Lyot Coronagraph design (left), pupil apodization techniques (top right) and nulling interferometric techniques (bottom righ).

\section{CORONAGRAPHIC APPROACHES}

A coronagraph must remove starlight while preserving, as much as possible, the light from off-axis sources (planets). This problem is made challenging by the small angular separation and large contrast between the star and the planet. High performance coronagraphs cannot simply rely on geometrical optics, but must instead use optical diffraction to remove starlight. Several approaches have been developed to do so, as shown in Figure 1.

Lyot coronagraphs rely on both a focal plane mask and a pupil plane mask to achieve starlight suppression. In the original Lyot coronagraph design [2], the focal plane mask is a small opaque disk, and the residual light in the pupil plane is concentrated around the edges of the pupil, which are blocked by a pupil mask (the Lyot mask). While the orginal Lyot coronagraph concept (using a circular opaque focal plane mask and a circular Lyot stop) cannot simultaneously reach high contrast at small angular separation, improvements to the original concept have led to many high performance coronagraph designs:

- By using focal plane masks including phase, Lyot coronagraph can be made to deliver high contrast much closer in to the optical axis than the original concept. This was first recognized by Roddier \& Roddier [3], who proposed using a phase-shifting focal plane mask in the Lyot coronagraph. This mask could be made very small (less than 1 1/D in diameter) and light passing through the mask would destructively interfere with light passing outside the mask within the geometric pupil. Other phase mask coronagraph designs include the 4-Quadrant Phase Mask [4], which uses a focal plane mask which shifts 2 out of 4 quadrants of the image by half a wave, the 8-octant coronagraph [5] 
which uses a larger number of sectors for improved tolerance to pointing errors and a deeper null on partially resolved sources, and the Optical Vortex coronagraph [6-9] which uses a continuous phase shifting pattern instead of discrete sectors, therefore avoiding the "dead zones" along the phase transitions in the focal plane.

- Amplitude apodization of the entrance pupil or focal plane mask can be used to tune the original Lyot design into a high performance coronagraph. In the Apodized Pupil Lyot Coronagraph (APLC) [10], the entrance pupil of the coronagraph is apodized and the Lyot stop is not undersized (its radius is the radius of the telescope pupil). In the Band-Limited Lyot Coronagraphs [11, 12], the focal plane mask is apodized to create perfectly (in the absence of aberrations) dark areas in the exit pupil, where the Lyot mask is designed to only transmit these dark areas. Both approaches can deliver very high contrast, but suffer from some loss in throughput and efficiency (light loss in the entrance pupil for the APLC; undersized Lyot stop for the band-limited coronagraphs).

A conceptually simpler approach to high contrast imaging is to modify the pupil shape (apodization) to avoid the presence of strond diffraction (Airy rings for example) in the focal plane, as shown in the upper right corner of Figure 1. In a conventional telescope, the Airy diffraction rings are due to the sharp edges of the pupil. If the entrance pupil of the telescope does not contain high spatial frequencies, it will not produce diffraction rings, and high contrast imaging is then possible without even requiring a focal plane mask. Apodization is performed by a pupil plane amplitude mask (Conventional Pupil Apodization - CPA), which can be a continuous mask [13-16] or a binary mask (also named shaped pupil) $[17,18]$, which are easier to manufacture. The pupil may also be apodized using phase instead of amplitude $[19,20]$, and the resulting PSF can allow high contrast imaging on one side of the image. Both amplitude and phase pupil apodization techniques become innefficient when designed for very high contrast, as most of the light entering the telescope is either absorbed by the apodization or diffracted outside the PSF core (producing an image in which only a small fraction of the planet light is concentrated in a diffraction peak), and the angular resolution of the telescope is not preserved. A higher efficiency alternative to these concepts is Phase-Induced Amplitude Apodization (PIAA), which produces the desired amplitude apodization by geometrical redistribution of the light in the pupil plane rather than selective absorption [21, 22]. Apodization is then performed by a set of (at least two) aspheric optics (mirror or lenses). PIAA is a high performance coronagraph offering simultaneoulsy high contrast, full throughput, full angular resolution and ability to image high contrast companions very close to the optical axis.

Interferometric coronagraphs (bottom right of Figure 1) look much like nulling interferometers: they rely on interferometric combination of discrete beams derived from the entrance pupil. At least two copies of the input telescope beam are produced, and then nulled in an interferometer. The two copies are identical for an on-axis point source (therefore producing a null at the output of the interferometer), but they are made to be different for an off-axis source (required to allow transmission of planet light through the nulling interferometer). In the Achromatic Interferometric Coronagraph (AIC) [23-26], one of the copies is rotated by $180 \mathrm{deg}$ prior to nulling. In the visible nuller [27], a translation of the wavefront is used instead. Both operations will preserve the on-axis wavefront but will introduce a difference between the two copies for off-axis sources.

Several coronagraphs lie at the boundary between the Lyot Coronagraph and Apodization groups identied in this section. This is due to the large performance improvement that pupil apodization can bring to the Lyot Coronagraph, as detailed in the description of the Apodized Pupil Lyot Coronagraph (APLC) concept [10]. A good example of advantageous combination between the two groups is the Phase-Induced Amplitude Apodization Complex Mask Coronagraph (PIAACMC). It offers very high coronagraphic performance [28] by combining the advantages of the phase mask Lyot coronagraph (ability to use a small size focal plane mask), the APLC (higher contrast achievable in a Lyot coronagraph by apodizing the telescope entrance aperture) and PIAA (lossless apodization). 
Point source / Radially averaged throughput

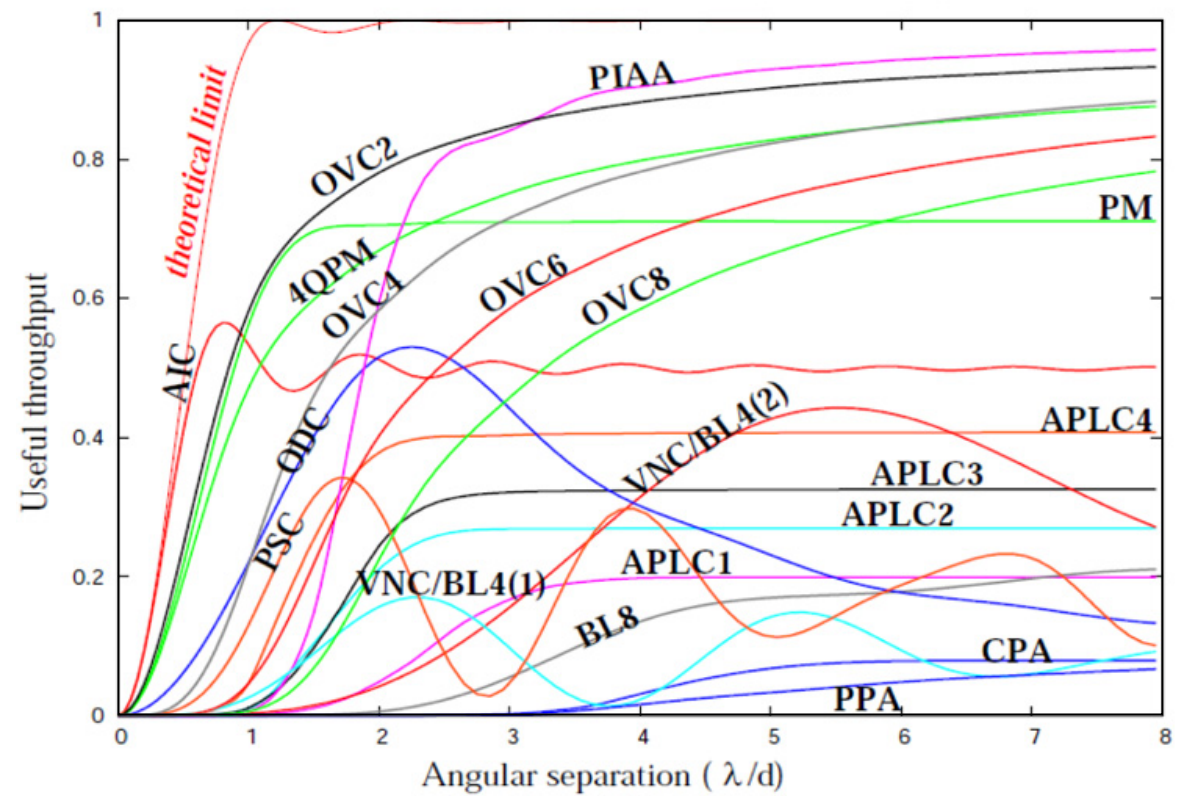

Figure 2. Coronagraph performance at 1e-10 contrast around a point source [29]. The highest performance coronagraphs are the Achromatic Interferometric Coronagraph (AIC), the Optical Vortex Coronagraph with topological charge $=2(\mathrm{OCV} 2)$, the Phase-Induced Amplitude Apodization (PIAA) and the 4 quadrants phase mask (4QPM)

\section{CORONAGRAPHIC PERFORMANCE}

Coronagraphs must provide high contrast image of the immediate surrounding of nearby starts while maintaining high efficiency. Metrics commonly used to describe coronagraph performance provide a quantitative assessment of how well coronagraphs can perform for different science cases. The most important metrics are:

- Raw contrast is a measure of how well a coronagraph can remove starlight in some areas of the image (where faint sources are to be detected). It is equal to the brightness of a companion (ratioed to the brightness of the central star) which would produce in the area of interest the same level of surface brightness as is left by the coronagraph.

- The Inner Working Angle (IWA) is the smallest angular separation at which a faint companion can be detected. For coronagraphs where residual starlight is below the desired contrast level, it is usually defined as the angular separation at which a companion's total transmitted light is half of the maximum transmitted light.

- The throughput (and discovery angle when appropriate) is the fraction of the companion's light transmitted by the coronagraph, and must be high to allow detection and spectroscopic characterization of exoplanets, which are usually faint targets.

Contrast, IWA and throughput are not independent metrics, and are linked together: IWA is given for a contrast value, and the IWA definition is using throughput. These metrics can be misleading when attempting to compare coronagraph performances. For example, a coronagraph with a $10 \%$ maximum throughput achieving 5\% throughput at $1 \lambda / \mathrm{d}$ has a smaller IWA than a coronagraph with a $100 \%$ maximum throughput achieving $30 \%$ throughput at $1 \lambda / \mathrm{d}$. A more accurate representation of coronagraph performance is given by the useful throughput definition for a given contrast. This quantity 


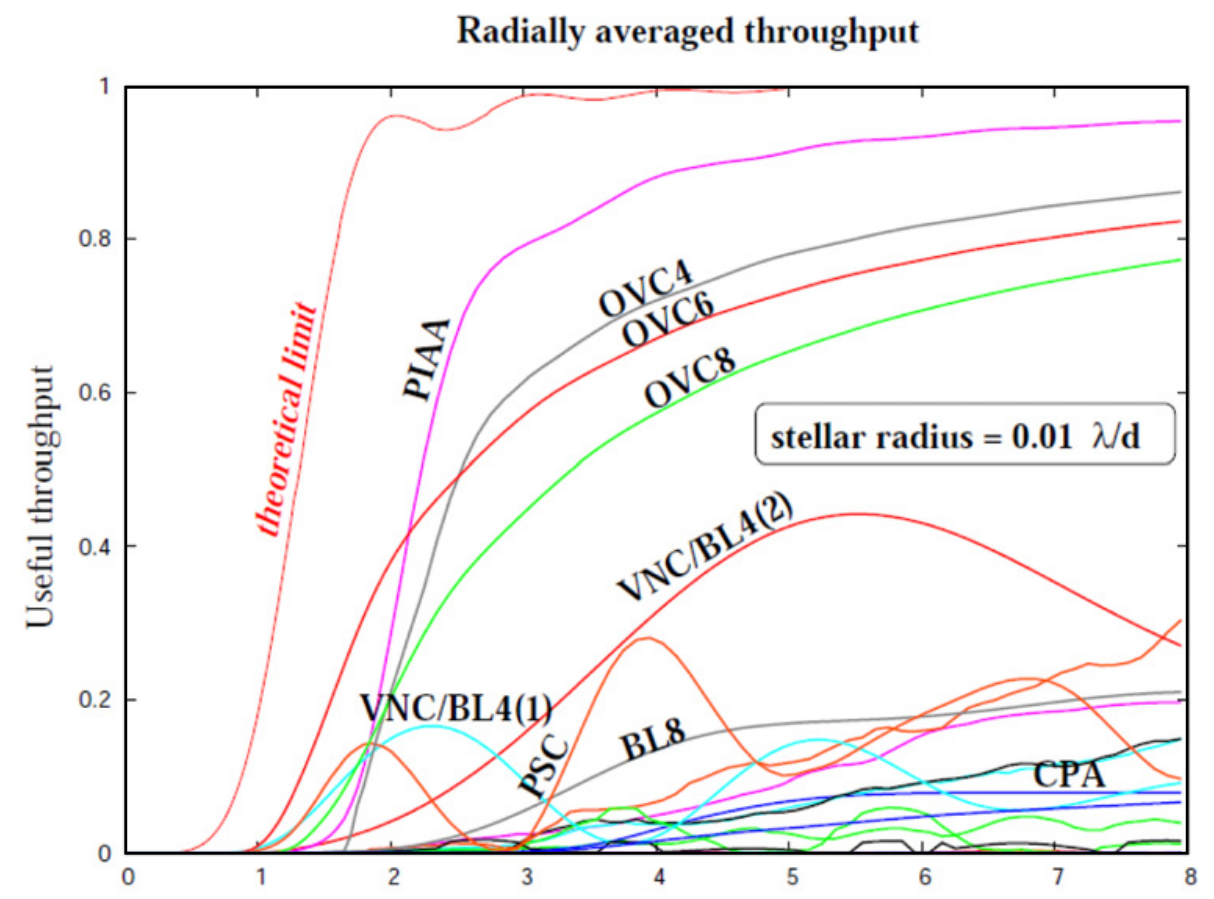

Figure 3. Coronagraph performance at $1 \mathrm{e}-10$ contrast around a point source. The highest performance coronagraphs are the Phase-Induced Amplitude Apodization (PIAA) and the Optical Vortex with topological charges 4 and 6 (OVC4, OVC6).

measures, for a given contrast, how much of the companion's light entering the telescope can be used toward detection, and can be plotted as a function of angular separation. A detailed description of how this metric is computed is given in a previous paper [29].

The useful throughput curves at 1e-10 contrast are given in Figure 2 for a large number of coronagraph concept for high contrast imaging around a point source. The same curves are shown in Figure 3 when accounting for a stellar angular size (radius) of $0.01 \lambda / \mathrm{d}$. Both figures are taken from a previous analysis of coronagraph performances [29], performed in year 2006.

Figures 2 and 3 show some important results for coronagraphy at the contrast level required for direct imaging of Earth-like planets:

- Both figures show a "theoretical limit" curve which is derived from fundamental principles (linearity of the optical system response in complex amplitude). Several high performance coronagraph concepts achieve performance close to the theoretical limit. This is especially true in the point source case.

- The coronagraph performances are very different at high contrast when the stellar angular size is taken into account. The theoretical limit performance is greatly reduced, and the coronagraphs which offer the best performance in the point source case are too sensitive to stellar angular size when it is taken into account.

- Once stellar angular size is taken into account, the best coronagraph choices for high contrast work appear to be the PIAA and Optical Vortex.

The newly developped PIAA complex mask coronagraph (PIAACMC) concept [28] offers a significant performance improvement over the baseline PIAA concept adopted for Figures 2 and 3, and is very close to the theoretical performance curves. It achieves its high performance by combining the advantages of the APLC (performance enhancement of a Lyot coronagraph thanks to apodization of the entrance 
pupil), the phase mask coronagraph (use of a phase-shifting focal plane mask to improve IWA) and PIAA (lossless apodization). In the point source case, a PIAACMC with a purely phase-shifting focal plane mask offers performance almost at the theoretical limit shown in Figure 2. With a larger partially transmissive phase-shifting focal plane mask, the PIAACMC can be optimized for partially resolved sources, and delivers performance close to the theoretical limit identified in figure 3 .

For ground-based high contrast imaging systems operating at more modest contrast (see section 4), a wider range of coronagraph concepts offers nearly ideal performance, and the effect of stellar angular size is more modest. The theoretical limit curve shown in Figure 2 does not have a strong dependence on contrast, and is almost the same, in the point source case, for the $\sim 1$ e- 6 ground-based contrast requirement as it is for the 1e-10 space-based contrast required for direct imaging of Earth-like planets.

\section{CONCLUSION}

While coronagraph testing in laboratories have reached very high contrast level (up to 1e9 contrast in vacuum tests), current ground-based are strongly limited by residual wavefront aberrations, and most of the advances in coronagraphic performance are expected to come from improvement in wavefront sensing and calibration techniques. Control of low-order aberrations is especially critical as those are the aberrations that limit coronagraphic performance and impose limits on the achievable inner working angle.

On ground-based observatories, the upcoming Extreme-AO systems on large telescopes, combining for the first time high performance adaptive optics with new coronagraph technologies, will greatly improve on-sky coronagraphic performance. Space coronagraphy, which is ultimately aimed at high contrast imaging for direct detection and characterization of potentially habitable planets, will also require high efficiency adaptive optics systems, although they will operate much slower. Despite these differences, both applications require the same combination of high performance coronagraphy and efficient adaptive optics correction.

\section{References}

[1] Extrasolar Planets Encyclopedia, http://exoplanet.eu/

[2] B. Lyot 1939, MNRAS, 99, 580

[3] F. Roddier \& C. Roddier 1997, PASP, 109, 815

[4] D. Rouan, P. Riaud, A. Bocaletti, Y. Clenet, \& A. Labeyrie 2000, PASP, 112, 1479

[5] N. Murakami, R. Uemura, N. Baba, J. Nishikawa, M. Tamura, N. Hashimoto, \& L. Abe, 2008, PASP, 120, 1112

[6] D. M. Palacios 2005, Proc. SPIE, 5905, 196

[7] G. Foo, D. M. Palacios, \& G. A. Swartzlander 2005, Opt. Lett., 30, 3308

[8] D. Mawet, P. Riaud, O. Absil, \& J. Surdej 2005, ApJ, 633, 1191

[9] G. A. Swartzlander, E. Ford, A-M. Rukiah, L. Close, M-A. Peters, D. M. Palacios, \& D. W. Wilson. 2008, Optics Express, 16, 10200

[10] R. Soummer, C. Aime, \& P. E. Fallon 2003, A\&A, 397, 1161

[11] M. J. Kuchner, \& W. A. Traub 2002, ApJ, 570, 900

[12] M. J. Kuchner, J. Crepp, J. Ge 2005, ApJ, 628, 466

[13] P. Jacquinot, \& B. Roisin-Dossier 1964, Prog. Opt., 3, 29

[14] P. Nisenson, \& C. Papaliolios 2001, ApJ, 548, L201

[15] R. Gonsalves, \& P. Nisenson 2003, PASP, 115, 706

[16] C. Aime 2005, A\&A, 434, 785

[17] N. J. Kasdin, R. J. Vanderbei, D. N. Spergel, \& M. G. Littman 2003, ApJ, 582, 1147

[18] R. J. Vanderbei, D. N. Spergel, \& N. J. Kasdin 2003, ApJ, 590, 593 
[19] J. L. Codona, \& R. Angel 2004, ApJ, 604, L117

[20] W. Yang, \& A.B. Kostinski 2004, ApJ, 605892

[21] O. Guyon 2003, A\&A, 404, 379

[22] O. Guyon, E. A. Pluzhnik, R. Galicher, F. Martinache, S. T. Ridgway, \& R. A. Woodruff 2005, ApJ, 622, 744

[23] J. Gay, \& Y. Rabbia 1996, C.R Acad. Sci. Paris, 322, 265

[24] P. Baudoz, Y. Rabbia, \& J. Gay 2000, A\&AS, 141, 319

[25] P. Baudoz, A. Boccaletti, Y. Rabbia, \& J. Gay 2005, PASP, 117, 1004

[26] A. V. Tavrov, Y. Kobayashi, Y. Tanaka, T. Shioda, Y. Otani, \& T. Kurokawa 2005, Optics Letters, 30, 2224

[27] B. P. Mennesson, M. Shao, B. M. Levine, J. K. Wallace, D. T. Liu, E. Serabyn, S. C. Unwin, \& C. A. Beichman 2003, Proc. SPIE, 4860, 32

[28] O. Guyon, F. Martinache, R. Belikov, \& R. Soummer 2010, ApJS, 190, 220

[29] O. Guyon, E. A. Pluzhnik, M. J. Kuchner, B. Collins, \& S. T. Ridgway 2006, ApJS, 167, 81 